\title{
MEAN CONVERGENCE THEOREMS AND WEAK LAWS OF LARGE NUMBERS FOR DOUBLE ARRAYS OF RANDOM ELEMENTS IN BANACH SPACES
}

\author{
Le Van Dung and Nguyen Duy Tien
}

\begin{abstract}
For a double array of random elements $\left\{V_{m n} ; m \geq 1, n \geq\right.$ $1\}$ in a real separable Banach space, some mean convergence theorems and weak laws of large numbers are established. For the mean convergence results, conditions are provided under which $k_{m n}^{-\frac{1}{r}} \sum_{i=1}^{u_{m}} \sum_{j=1}^{v_{n}}\left(V_{i j}-\right.$ $\left.E\left(V_{i j} \mid \mathcal{F}_{i j}\right)\right) \rightarrow 0$ in $L_{r}(0<r<2)$. The weak law results provide conditions for $k_{m n}^{-\frac{1}{r}} \sum_{i=1}^{T_{m}} \sum_{j=1}^{\tau_{n}}\left(V_{i j}-E\left(V_{i j} \mid \mathcal{F}_{i j}\right)\right) \rightarrow 0$ in probability where $\left\{T_{m} ; m \geq 1\right\}$ and $\left\{\tau_{n} ; n \geq 1\right\}$ are sequences of positive integer-valued random variables, $\left\{k_{m n} ; m \geq 1, n \geq 1\right\}$ is an array of positive integers. The sharpness of the results is illustrated by examples.
\end{abstract}

\section{Introduction}

The classical notion of uniform integrability of a sequence $\left\{X_{n} ; n \geq 1\right\}$ of integrable random variables is defined through the condition

$$
\lim _{a \rightarrow \infty} \sup _{n \geq 1} E\left|X_{n}\right| I\left(\left|X_{n}\right|>a\right)=0 .
$$

Landers and Rogge [4] prove that the uniform integrability condition is sufficient in order that a sequence of pairwise independent random variables verifies the weak law of large numbers.

Chandra [2] obtains the weak law of large numbers under the condition which is weaker than uniform integrability: the condition of Cesàro uniform integrability. A sequence $\left\{X_{n} ; n \geq 1\right\}$ of integrable random variables is said to be Cesàro uniformly integrable if

$$
\lim _{a \rightarrow \infty} \sup _{n \geq 1} \frac{1}{k_{n}} \sum_{j=1}^{k_{n}} E\left|X_{j}\right| I\left(\left|X_{j}\right|>a\right)=0,
$$

where $\left\{k_{n} ; n \geq 1\right\}$ is a sequence of positive integers such that $\lim _{n \rightarrow \infty} k_{n}=\infty$.

Received August 8, 2008; Revised August 25, 2009.

2000 Mathematics Subject Classification. 60B11, 60B12, 60F15, 60F25, 60G42.

Key words and phrases. martingale type $p$ Banach spaces, double arrays of random elements, weighted double sums, weak laws of large numbers, mean convergence theorem. 
Definition. Let $\left\{X_{n} ; n \geq 1\right\}$ be a sequence of random variables and $\left\{a_{n j} ; 1 \leq\right.$ $\left.j \leq v_{n}, n \geq 1\right\}$ be an array of constants with $\sum_{j=1}^{v_{n}}\left|a_{n j}\right| \leq C$ for all $n \in \mathbb{N}$ and some constant $C>0$. The sequence $\left\{X_{n} ; n \geq 1\right\}$ is $\left\{a_{n j}\right\}$-uniform integrable if

$$
\lim _{a \rightarrow \infty} \sup _{n \geq 1} \sum_{j=1}^{v_{n}}\left|a_{n j}\right| E\left|X_{j}\right| I\left(\left|X_{j}\right|>a\right)=0,
$$

where $\left\{v_{n} ; n \geq 1\right\}$ is a sequence of positive integers such that $\lim _{n \rightarrow \infty} v_{n}=\infty$.

Under the condition of $\left\{a_{n j}\right\}$-uniform integrability, Ordóñez Cabrera [5] obtains the weak law of large numbers for weighted sums of pairwise independent random variables; the condition of pairwise independence can be even dropped, at the price of slightly strengthening the conditions on the weights. Recently, Thanh [10] obtains the mean convergence theorems for the weighted sums $\sum_{i=1}^{k_{m}} \sum_{j=1}^{l_{n}} a_{m n i j}\left(X_{i j}-E X_{i j}\right)$ in $L_{p}$ and the weak laws of large numbers with random indices for the weighted sums $\sum_{i=1}^{T_{m}} \sum_{j=1}^{\tau_{n}} a_{m n i j}\left(X_{i j}-E X_{i j}\right)$, where $\left\{X_{i j}, i \geq 1, j \geq 1\right\}$ is an array of random variable, $\left\{a_{m n i j} ; m, n, i, j \geq 1\right\}$ are constants, $\left\{T_{m} ; m \geq 1\right\}$ and $\left\{\tau_{n} ; n \geq 1\right\}$ are sequences of positive integervalued random variables.

Sung [9] introduces the concept of Cesàro type uniform integrability with exponent $r$.

Definition. Let $\left\{X_{n} ; n \geq 1\right\}$ be a sequence of random variables and $r>0$. The array $\left\{X_{n} ; n \geq 1\right\}$ is said to be Cesàro type uniformly integrable with exponent $r$ if

$$
\sup _{n \geq 1} \frac{1}{k_{n}} \sum_{j=1}^{v_{n}} E\left|X_{j}\right|^{r}<\infty \text { and } \lim _{a \rightarrow \infty} \sup _{n \geq 1} \frac{1}{k_{n}} \sum_{j=1}^{v_{n}} E\left|X_{j}\right|^{r} I\left(\left|X_{j}\right|>a\right)=0,
$$

where $\left\{k_{n} ; n \geq 1\right\}$ and $\left\{v_{n} ; n \geq 1\right\}$ are two sequences of positive integers such that $\lim _{n \rightarrow \infty} \bar{k}_{n}=\lim _{n \rightarrow \infty} v_{n}=\infty$.

In this paper, we not only enlarge on some results of Adler et al. [1] and Thanh [10] but we also weaken the suppositions and bring more general results.

\section{Preliminaries}

For $a, b \in \mathbb{R}, \min \{a, b\}, \max \{a, b\}$ and the integer part of $a$ will be denoted, respectively, by $a \wedge b, a \vee b$ and $[a]$. Throughout this paper, the symbol $C$ will denote a generic constant $(0<C<\infty)$ which is not necessarily the same one in each appearance.

Technical definitions relevant to the current work will be discussed in this section. Scalora [8] introduced the idea of the conditional expectation of a random element in a Banach space. For a random element $V$ and sub $\sigma$ algebra $\mathcal{G}$ of $\mathcal{F}$, the conditional expectation $E(V \mid \mathcal{G})$ is defined analogously to that in the random variable case and enjoys similar properties. 
A real separable Banach space $\mathcal{X}$ is said to be martingale type $p(1 \leq p \leq 2)$ if there exists a finite positive constant $C$ such that for all martingales $\left\{S_{n} ; n \geq 1\right\}$ with values in $\mathcal{X}$

$$
\sup _{n \geq 1} E\left\|S_{n}\right\|^{p} \leq C \sum_{n=1}^{\infty} E\left\|S_{n}-S_{n-1}\right\|^{p} .
$$

It can be shown using classical methods from martingale theory that if $\mathcal{X}$ is of martingale type $p$, then for all $1 \leq r<\infty$ there exists a finite constant $C$ such that

$$
E \sup _{n \geq 1}\left\|S_{n}\right\|^{r} \leq C E\left(\sum_{n=1}^{\infty}\left\|S_{n}-S_{n-1}\right\|^{p}\right)^{\frac{r}{p}} .
$$

Clearly every real separable Banach space is of martingale type 1 and the real line (the same as any Hilbert space) is of martingale type 2. If a real separable Banach space of martingale type $p$ for some $1<p \leq 2$, then it is of martingale type $r$ for all $r \in[1, p)$.

It follows from the Hoffmann-Jørgensen and Pisier [3] characterization of Rademacher type $p$ Banach spaces that if a Banach space is of martingale type $p$, then it is of Rademacher type $p$. But the notion of martingale type $p$ is only superficially similar to that of Rademacher type $p$ and has a geometric characterization in terms of smoothness. For proofs and more details, the reader may refer to Pisier $[6,7]$.

The following lemma is needed to prove our main results.

Lemma 2.1. Suppose that the array of random elements $\left\{V_{m n} ; m \geq 1, n \geq 1\right\}$ is Cesàro type uniform integrability with exponent $r$, in the sense that

$$
\sup _{m \geq 1, n \geq 1} \frac{1}{k_{m n}} \sum_{i=1}^{u_{m}} \sum_{j=1}^{v_{n}} E\left\|V_{i j}\right\|^{r} \leq M<\infty
$$

and

$$
\lim _{a \rightarrow \infty} \sup _{m \geq 1, n \geq 1} \frac{1}{k_{m n}} \sum_{i=1}^{u_{m}} \sum_{j=1}^{v_{n}} E\left\|V_{i j}\right\|^{r} I\left(\left\|V_{i j}\right\|^{r}>a\right)=0,
$$

where $\left\{k_{m n} ; m \geq 1, n \geq 1\right\}$ is an array of positive integers such that

$$
\lim _{m \vee n \rightarrow \infty} k_{m n}=\infty \text {. }
$$

Then

$$
\frac{1}{k_{m n}^{\frac{\beta}{r}}} \sum_{i=1}^{u_{m}} \sum_{j=1}^{v_{n}} E\left\|V_{i j}\right\|^{\beta} I\left(\left\|V_{i j}\right\|^{r} \leq k_{m n}\right) \rightarrow 0 \text { as } m \vee n \rightarrow \infty \text { if } r<\beta
$$

Proof.

$$
\frac{1}{k_{m n}^{\frac{\beta}{r}}} \sum_{i=1}^{u_{m}} \sum_{j=1}^{v_{n}} E\left\|V_{i j}\right\|^{\beta} I\left(\left\|V_{i j}\right\|^{r} \leq k_{m n}\right)
$$




$$
\begin{aligned}
= & \frac{1}{k_{m n}^{\frac{\beta}{r}}} \sum_{i=1}^{u_{m}} \sum_{j=1}^{v_{n}} \sum_{l=1}^{k_{m n}} E\left\|V_{i j}\right\|^{\beta} I\left(l-1<\left\|V_{i j}\right\|^{r} \leq l\right) \\
\leq & \frac{1}{k_{m n}^{\frac{\beta}{r}}} \sum_{i=1}^{u_{m}} \sum_{j=1}^{v_{n}} \sum_{l=1}^{k_{m n}} l^{\frac{\beta-r}{r}} E\left\|V_{i j}\right\|^{r} I\left(l-1<\left\|V_{i j}\right\|^{r} \leq l\right) \\
= & \frac{1}{k_{m n}^{\frac{\beta}{r}}} \sum_{i=1}^{u_{m}} \sum_{j=1}^{v_{n}} \sum_{l=1}^{k_{m n}} l^{\frac{\beta-r}{r}}\left(E\left\|V_{i j}\right\|^{r} I\left(\left\|V_{i j}\right\|^{r}>l-1\right)-E\left\|V_{i j}\right\|^{r} I\left(\left\|V_{i j}\right\|^{r}>l\right)\right) \\
\leq & \frac{1}{k_{m n}^{\frac{\beta}{r}}} \sum_{i=1}^{u_{m}} \sum_{j=1}^{v_{n}} E\left\|V_{i j}\right\|^{r} I\left(\left\|V_{i j}\right\|^{r}>0\right) \\
& +\frac{1}{k_{m n}^{\frac{\beta}{r}}} \sum_{i=1}^{u_{m}} \sum_{j=1}^{v_{n}} \sum_{l=1}^{k_{m n}-1}\left((l+1)^{\frac{\beta-r}{r}}-(l)^{\frac{\beta-r}{r}}\right) E\left\|V_{i j}\right\|^{r} I\left(\left\|V_{i j}\right\|^{r}>l\right) \\
= & : A_{m n}+B_{m n} .
\end{aligned}
$$

For $A_{m n}$ we have

$$
A_{m n} \leq \frac{1}{k_{m n}^{\frac{\beta}{r}-1}} \sup _{m \geq 1, n \geq 1} \frac{1}{k_{m n}} \sum_{i=1}^{u_{m}} \sum_{j=1}^{v_{n}} E\left\|V_{i j}\right\|^{r} \rightarrow 0 \quad \text { as } \quad m \vee n \rightarrow \infty
$$

by $\frac{\beta}{r}-1>0$ and $(2.2)$. Next, we will show that

$$
\lim _{m \vee n \rightarrow \infty} B_{m n}=0 .
$$

For arbitrary $\varepsilon>0$, since (2.3) there exists $a_{0}$ such that

$$
\sup _{m \geq 1, n \geq 1} \frac{1}{k_{m n}} \sum_{i=1}^{u_{m}} \sum_{j=1}^{v_{n}} E\left\|V_{i j}\right\|^{r} I\left(\left\|V_{i j}\right\|^{r}>a_{0}\right)<\frac{\varepsilon}{2} .
$$

Now for all large enough $m \vee n$, we have $k_{m n} \geq\left(\left[a_{0}\right]+1\right)\left(\frac{2 M}{\varepsilon}\right)^{\frac{r}{\beta-r}}$ and

$$
\begin{aligned}
& B_{m n} \\
= & \frac{1}{k_{m n}^{\frac{\beta}{r}}} \sum_{i=1}^{u_{m}} \sum_{j=1}^{v_{n}} \sum_{l=1}^{\left[a_{0}\right]}\left((l+1)^{\frac{\beta-r}{r}}-(l)^{\frac{\beta-r}{r}}\right) E\left\|V_{i j}\right\|^{r} I\left(\left\|V_{i j}\right\|^{r}>l\right) \\
& +\frac{1}{k_{m n}^{\frac{\beta}{r}}} \sum_{i=1}^{u_{m}} \sum_{j=1}^{v_{n}} \sum_{l=\left[a_{0}\right]+1}^{k_{m n}-1}\left((l+1)^{\frac{\beta-r}{r}}-(l)^{\frac{\beta-r}{r}}\right) E\left\|V_{i j}\right\|^{r} I\left(\left\|V_{i j}\right\|^{r}>l\right) \\
\leq & \frac{1}{k_{m n}^{\frac{\beta}{r}}} \sum_{i=1}^{u_{m}} \sum_{j=1}^{v_{n}} \sum_{l=1}^{\left[a_{0}\right]}\left((l+1)^{\frac{\beta-r}{r}}-(l)^{\frac{\beta-r}{r}}\right) E\left\|V_{i j}\right\|^{r} I\left(\left\|V_{i j}\right\|^{r}>1\right)
\end{aligned}
$$




$$
\begin{aligned}
& +\frac{1}{k_{m n}^{\frac{\beta}{r}}} \sum_{i=1}^{u_{m}} \sum_{j=1}^{v_{n}} \sum_{l=\left[a_{0}\right]+1}^{k_{m n}-1}\left((l+1)^{\frac{\beta-r}{r}}-(l)^{\frac{\beta-r}{r}}\right) E\left\|V_{i j}\right\|^{r} I\left(\left\|V_{i j}\right\|^{r}>\left[a_{0}\right]+1\right) \\
= & \frac{1}{k_{m n}^{\frac{\beta}{r}}} \sum_{i=1}^{u_{m}} \sum_{j=1}^{v_{n}}\left(\left(\left[a_{0}\right]+1\right)^{\frac{\beta-r}{r}}-1\right) E\left\|V_{i j}\right\|^{r} I\left(\left\|V_{i j}\right\|^{r}>1\right) \\
& +\frac{1}{k_{m}^{\frac{\beta}{r}}} \sum_{i=1}^{u_{m}} \sum_{j=1}^{v_{n}}\left(\left(k_{m n}\right)^{\frac{\beta-r}{r}}-\left(\left[a_{0}\right]+1\right)^{\frac{\beta-r}{r}}\right) E\left\|V_{i j}\right\|^{r} I\left(\left\|V_{i j}\right\|^{r}>\left[a_{0}\right]+1\right) \\
\leq & \left(\frac{\left[a_{0}\right]+1}{k_{m n}}\right)^{\frac{\beta-r}{r}} \sup _{m \geq 1, n \geq 1} \frac{1}{k_{m n}} \sum_{i=1}^{u_{m}} \sum_{j=1}^{v_{n}} E\left\|V_{i j}\right\|^{r} \\
& +\sup _{m \geq 1, n \geq 1} \frac{1}{k_{m n}} \sum_{i=1}^{u_{m}} \sum_{j=1}^{v_{n}} E\left\|V_{i j}\right\|^{r} I\left(\left\|V_{i j}\right\|^{r}>\left[a_{0}\right]+1\right)<\frac{\varepsilon}{2}+\frac{\varepsilon}{2}=\varepsilon .
\end{aligned}
$$

Thus $B_{m n} \rightarrow 0$ as $m \vee n \rightarrow \infty$. The proof is completed.

To prove Lemma 2.3, we need the following lemma:

Lemma 2.2. If $\left\{X_{k l}, \mathcal{F}_{l} ; l \geq 1\right\}, k=1,2, \ldots, m$ are nonnegative submartingales, then $\left\{\max _{1 \leq k \leq m} X_{k l}, \mathcal{F}_{l} ; l \geq 1\right\}$ is a nonnegative submartingale.

Proof. For $L>l \geq 1$,

$$
E\left(\max _{1 \leq k \leq m} X_{k L} \mid \mathcal{F}_{l}\right) \geq \max _{1 \leq k \leq m} E\left(X_{k L} \mid \mathcal{F}_{l}\right) \geq \max _{1 \leq k \leq m} X_{k l} .
$$

Let $\mathcal{F}_{k l}$ be the $\sigma$-field generated by the family of random elements $\left\{V_{i j} ; i<\right.$ $k$ or $j<l\}, \mathcal{F}_{1,1}=\{\emptyset ; \Omega\}$. We have the following lemma:

Lemma 2.3. Let $\left\{V_{i j} ; 1 \leq i \leq m, 1 \leq j \leq n\right\}$ be a collection of $m n$ random elements in a martingale type $p(1 \leq p \leq 2)$ Banach space with $E\left(V_{i j} \mid \mathcal{F}_{i j}\right)=0$ for all $i, j(1 \leq i \leq m, 1 \leq j \leq n)$. Then

$$
\begin{aligned}
& E\left\|\sum_{i=1}^{m} \sum_{j=1}^{n} V_{i j}\right\|^{p} \leq C \sum_{i=1}^{m} \sum_{j=1}^{n} E\left\|V_{i j}\right\|^{p} ; \\
& P\left\{\max _{\substack{1 \leq k \leq m \\
1 \leq l \leq n}}\left\|\sum_{i=1}^{k} \sum_{j=1}^{l} V_{i j}\right\|>\varepsilon\right\} \leq \frac{C}{\varepsilon^{p}} \sum_{i=1}^{m} \sum_{j=1}^{n} E\left\|V_{i j}\right\|^{p} \quad \forall \varepsilon>0 .
\end{aligned}
$$

Proof. First, we prove (2.3). Set $S_{k n}=\sum_{i=1}^{k} \sum_{j=1}^{n} V_{i j}$, since $E\left(V_{i j} \mid \mathcal{F}_{i j}\right)=0$ we have that $\left\{S_{k n}, \mathcal{G}_{k}=\mathcal{F}_{k+1,1} ; 1 \leq k \leq m\right\}$ is a martingale. Thus

$$
E\left\|\sum_{i=1}^{m} \sum_{j=1}^{n} V_{i j}\right\|^{p} \leq E \max _{1 \leq k \leq m}\left\|S_{k n}\right\|^{p} \leq C \sum_{k=1}^{m} E\left\|\sum_{j=1}^{n} V_{k j}\right\|^{p} .
$$


On the other hand, for each $k(1 \leq k \leq m),\left\{\sum_{j=1}^{l} V_{k j}, \mathcal{G}_{k l}=\mathcal{F}_{k, l+1} ; 1 \leq l \leq n\right\}$ is a martingale. Hence,

$$
E\left\|\sum_{j=1}^{n} V_{k j}\right\|^{p} \leq E \max _{1 \leq l \leq n}\left\|\sum_{j=1}^{l} V_{k j}\right\|^{p} \leq C \sum_{l=1}^{n} E\left\|V_{k l}\right\|^{p} .
$$

Combining (2.5) and (2.6) yields the conclusion (2.3).

Next, we will prove (2.4). Set $S_{k l}=\sum_{i=1}^{k} \sum_{j=1}^{l} V_{i j}, Y_{l}=\max _{1 \leq k \leq m}\left\|S_{k l}\right\|$. If $\sigma_{l}$ is a $\sigma$-field generated by $\left\{V_{i j} ; 1 \leq i \leq m, 1 \leq j \leq l\right\}$, then for each $l(1 \leq l \leq n), \sigma_{l} \subset \mathcal{F}_{i, l+1}$ for all $i \geq 1$, which follows that $E\left(V_{i, l+1} \mid \sigma_{l}\right)=$ $E\left(E\left(V_{i, l+1} \mid \mathcal{F}_{i, l+1}\right) \mid \sigma_{l}\right)=0$. Thus, we have

$$
E\left(S_{k, l+1} \mid \sigma_{l}\right)=E\left(S_{k l} \mid \sigma_{l}\right)+\sum_{i=1}^{k} E\left(V_{i, l+1} \mid \sigma_{l}\right)=S_{k l} .
$$

It means that $\left\{S_{k l}, \sigma_{l} ; 1 \leq l \leq n\right\}$ is a martingale. Hence, $\left\{\left\|S_{k l}\right\|, \sigma_{l} ; 1 \leq l \leq\right.$ $n\}$ is a nonnegative submartingale for each $k=1,2, \ldots, m$, which follows by Lemma 2.1 that $\left\{Y_{l}, \sigma_{l} ; 1 \leq l \leq n\right\}$ is a nonnegative submartingale. Applying Kolmogorov's inequality, we obtain

$$
\begin{aligned}
P\left\{\max _{\substack{1 \leq k \leq m \\
1 \leq l \leq n}}\left\|\sum_{i=1}^{k} \sum_{j=1}^{l} V_{i j}\right\|>\varepsilon\right\} & =P\left\{\max _{1 \leq l \leq n} Y_{l}>\varepsilon\right\} \leq \frac{1}{\varepsilon^{p}} E Y_{n}^{p} \\
& =\frac{1}{\varepsilon^{p}} E \max _{1 \leq k \leq m}\left\|S_{k n}\right\|^{p} \leq \frac{C}{\varepsilon^{p}} \sum_{k=1}^{m} E\left\|\sum_{j=1}^{n} V_{k j}\right\|^{p} \\
& \leq \frac{C}{\varepsilon^{p}} \sum_{k=1}^{m} E \max _{1 \leq l \leq n}\left\|\sum_{j=1}^{l} V_{k j}\right\|^{p} \\
& \leq \frac{C}{\varepsilon^{p}} \sum_{k=1}^{m} \sum_{l=1}^{n} E\left\|V_{k l}\right\|^{p} \\
& =\frac{C}{\varepsilon^{p}} \sum_{i=1}^{m} \sum_{j=1}^{n} E\left\|V_{i j}\right\|^{p}
\end{aligned}
$$

The proof is completed.

\section{Main results}

With the preliminaries accounted for, the main results may now be established. In the following we let $\left\{V_{m n} ; m \geq 1, n \geq 1\right\}$ be an array of random elements defined on a probability $(\Omega, \mathcal{F}, P)$ and taking values in a real separable Banach space $\mathcal{X}$ with norm $\|\cdot\|, \mathcal{F}_{k l}$ be a $\sigma$-field generated by $\left\{V_{i j} ; i<\right.$ $k$ or $j<l\}, \mathcal{F}_{1,1}=\{\emptyset ; \Omega\}$. Let $\left\{u_{n} ; n \geq 1\right\},\left\{v_{n} ; n \geq 1\right\}$ be sequences of positive integers such that $\lim _{n \rightarrow \infty} u_{n}=\lim _{n \rightarrow \infty} v_{n}=\infty,\left\{k_{m n} ; m \geq 1, n \geq 1\right\}$ be an array of positive integers such that $\lim _{m \vee n \rightarrow \infty} k_{m n}=\infty$. 
Theorem 3.1. Let $1 \leq r<p \leq 2$, Banach space $\mathcal{X}$ be a martingale type $p$. Suppose that $\left\{V_{m n} ; m \geq 1, n \geq 1\right\}$ satisfies the Cesàro type uniform integrability with exponent $r$, in the sense that (2.1) and (2.2) hold. Then

$$
\frac{1}{k_{m n}^{\frac{1}{r}}} \sum_{i=1}^{u_{m}} \sum_{j=1}^{v_{n}}\left(V_{i j}-E\left(V_{i j} \mid \mathcal{F}_{i j}\right)\right) \rightarrow 0 \text { in } L_{r} \text { as } n \vee m \rightarrow \infty
$$

Proof. For each $m, n, 1 \leq i \leq u_{m}, 1 \leq j \leq v_{n}$, set

$$
\begin{gathered}
V_{m n i j}^{\prime}=V_{i j} I\left(\left\|V_{i j}\right\|^{r} \leq k_{m n}\right), V_{m n i j}^{\prime \prime}=V_{i j} I\left(\left\|V_{i j}\right\|^{r}>k_{m n}\right), \\
U_{m n i j}^{\prime}=E\left(V_{m n i j}^{\prime} \mid \mathcal{F}_{i j}\right), U_{m n i j}^{\prime \prime}=E\left(V_{m n i j}^{\prime \prime} \mid \mathcal{F}_{i j}\right) .
\end{gathered}
$$

Observe that for each $i$ and $j, 1 \leq i \leq u_{m}, 1 \leq j \leq v_{n}$, we have

$$
V_{i j}-E\left(V_{i j} \mid \mathcal{F}_{i j}\right)=\left(V_{m n i j}^{\prime}-U_{m n i j}^{\prime}\right)+\left(V_{m n i j}^{\prime \prime}-U_{m n i j}^{\prime \prime}\right)
$$

and $E\left(V_{m n i j}^{\prime}-U_{m n i j}^{\prime} \mid \mathcal{F}_{i j}\right)=E\left(V_{m n i j}^{\prime \prime}-U_{m n i j}^{\prime \prime} \mid \mathcal{F}_{i j}\right)=0$. Hence,

$$
\begin{aligned}
& E\left\|\frac{1}{k_{m n}^{\frac{1}{r}}} \sum_{i=1}^{u_{m}} \sum_{j=1}^{v_{n}} V_{i j}\right\|^{r} \\
& =\frac{1}{k_{m n}} E\left\|\sum_{i=1}^{u_{m}} \sum_{j=1}^{v_{n}}\left(V_{m n i j}^{\prime}-U_{m n i j}^{\prime}\right)+\sum_{i=1}^{u_{m}} \sum_{j=1}^{v_{n}}\left(V_{m n i j}^{\prime \prime}-U_{m n i j}^{\prime \prime}\right)\right\|^{r} \\
& \leq C \frac{1}{k_{m n}} E\left\|\sum_{i=1}^{u_{m}} \sum_{j=1}^{v_{n}}\left(V_{m n i j}^{\prime}-U_{m n i j}^{\prime}\right)\right\|^{r} \\
& +C \frac{1}{k_{m n}} E\left\|\sum_{i=1}^{u_{m}} \sum_{j=1}^{v_{n}}\left(V_{m n i j}^{\prime \prime}-U_{m n i j}^{\prime \prime}\right)\right\|^{r} \quad \text { (by } c_{r} \text {-inequality) } \\
& \leq C \frac{1}{k_{m n}}\left(E\left\|\sum_{i=1}^{u_{m}} \sum_{j=1}^{v_{n}}\left(V_{m n i j}^{\prime}-U_{m n i j}^{\prime}\right)\right\|^{p}\right)^{r / p} \\
& +C \frac{1}{k_{m n}} E\left\|\sum_{i=1}^{u_{m}} \sum_{j=1}^{v_{n}}\left(V_{m n i j}^{\prime \prime}-U_{m n i j}^{\prime \prime}\right)\right\|^{r} \quad \text { (by Liapunov's inequality) } \\
& \leq C \frac{1}{k_{m n}}\left(\sum_{i=1}^{u_{m}} \sum_{j=1}^{v_{n}} E\left\|\left(V_{m n i j}^{\prime}-U_{m n i j}^{\prime}\right)\right\|^{p}\right)^{r / p} \\
& +C \frac{1}{k_{m n}} \sum_{i=1}^{u_{m}} \sum_{j=1}^{v_{n}} E\left\|\left(V_{m n i j}^{\prime \prime}-U_{m n i j}^{\prime \prime}\right)\right\|^{r} \quad \text { (by Lemma 2.3) }
\end{aligned}
$$




$$
\begin{aligned}
\leq & C \frac{1}{k_{m n}}\left(\sum_{i=1}^{u_{m}} \sum_{j=1}^{v_{n}} E\left\|V_{i j}\right\|^{p} I\left(\left\|V_{i j}\right\|^{r} \leq k_{m n}\right)\right)^{r / p} \\
& +C \frac{1}{k_{m n}} \sum_{i=1}^{u_{m}} \sum_{j=1}^{v_{n}} E\left\|V_{i j}\right\|^{r} I\left(\left\|V_{i j}\right\|^{r}>k_{m n}\right) \quad \text { (by } c_{r} \text {-inequality) } \\
\leq & C\left(\frac{1}{k_{m n}^{\frac{p}{r}}} \sum_{i=1}^{u_{m}} \sum_{j=1}^{v_{n}} E\left\|V_{i j}\right\|^{p} I\left(\left\|V_{i j}\right\|^{r} \leq k_{m n}\right)\right)^{r / p} \\
& +C\left(\sup _{m \geq 1, n \geq 1} \frac{1}{k_{m n}} \sum_{i=1}^{u_{m}} \sum_{j=1}^{v_{n}} E\left\|V_{i j}\right\|^{r} I\left(\left\|V_{i j}\right\|^{r}>k_{m n}\right)\right) \rightarrow 0 \text { as } m \vee n \rightarrow \infty,
\end{aligned}
$$

by Lemma 2.1 with $\beta=p$ and (2.2). The proof is completed.

Corollary 3.2. Let $1 \leq r<p \leq 2$, Banach space $\mathcal{X}$ be a martingale type $p$. Let $\left\{A_{m n i j} ; m \geq 1, n \geq 1,1 \leq i \leq u_{m}, 1 \leq j \leq v_{n}\right\}$ be an array of random variables satisfying $\sum_{i=1}^{u_{m}} \sum_{j=1}^{v_{n}} E\left|A_{m n i j}\right|^{r} \leq C<\infty$ for all $m \geq 1, n \geq 1$. Suppose that $\left\{A_{m n i j} ; 1 \leq i \leq u_{m}, 1 \leq j \leq v_{n}\right\}$ and $\left\{V_{i j} ; i \geq 1, j \geq 1\right\}$ are independent for all $m \geq 1, n \geq 1$. Assume that the following conditions hold:

(i) $\left\{\left\|V_{m n}\right\|^{r} ; m \geq 1, n \geq 1\right\}$ is $\left\{E\left|A_{m n i j}\right|^{r}\right\}$-uniformly integrable, in the sense that

$$
\lim _{a \rightarrow \infty} \sup _{m \geq 1, n \geq 1} \sum_{i=1}^{u_{m}} \sum_{j=1}^{v_{n}} E\left\|A_{m n i j} V_{i j}\right\|^{r} I\left(\left\|V_{i j}\right\|>a\right)=0
$$

(ii) $\sup _{1 \leq i \leq u_{m}, 1 \leq j \leq v_{n}} E\left|A_{m n i j}\right| \rightarrow 0$ as $m \vee n \rightarrow \infty$.

Then

$$
\sum_{i=1}^{u_{m}} \sum_{j=1}^{v_{n}} A_{m n i j}\left(V_{i j}-E\left(V_{i j} \mid \mathcal{F}_{i j}\right)\right) \rightarrow 0 \text { in } L_{r} \text { as } m \vee n \rightarrow \infty
$$

Proof. Let

$$
k_{m n}=\left[\frac{1}{\sup _{1 \leq i \leq u_{m}, 1 \leq j \leq v_{n}} E\left|A_{m n i j}\right|}\right] .
$$

Then $k_{m n} \rightarrow \infty$ as $m \vee n \rightarrow \infty$. It is easy to prove that

$$
\sup _{m \geq 1, n \geq 1} \sum_{i=1}^{u_{m}} \sum_{j=1}^{v_{n}} E\left\|A_{m n i j} V_{i j}\right\|^{r}<\infty \text {. }
$$

Hence, take $k_{m n}^{1 / r} A_{m n i j} V_{i j}$ instead $V_{i j}$ in Theorem 3.1, we have by (i) that $\sup _{n \geq 1, m \geq 1} \frac{1}{k_{m n}} \sum_{i=1}^{u_{m}} \sum_{j=1}^{v_{n}} E\left\|k_{m n}^{1 / r} A_{m n i j} V_{i j}\right\|^{r}=\sup _{m \geq 1, n \geq 1} \sum_{i=1}^{u_{m}} \sum_{j=1}^{v_{n}} E\left\|A_{m n i j} V_{i j}\right\|^{r}<\infty$. 
On the other hand, for all large enough $m \vee n$ we have

$$
\sup _{1 \leq i \leq u_{m}, 1 \leq j \leq v_{n}} E\left|A_{m n i j}\right| \leq 1
$$

which follows that

$$
k_{m n} E\left|A_{m n i j}\right|^{r} \leq \frac{E\left|A_{m n i j}\right|^{r}}{\sup _{1 \leq i \leq u_{m}, 1 \leq j \leq v_{n}} E\left|A_{m n i j}\right|} \leq \frac{E\left|A_{m n i j}\right|^{r}}{\sup _{1 \leq i \leq u_{m}, 1 \leq j \leq v_{n}} E\left|A_{m n i j}\right|^{r}} \leq 1 .
$$

Therefore, for all large enough $m \vee n, k_{m n}\left|A_{m n i j}\right|^{r} \leq 1$ a.s.. Thus, it follows by (i) that

$$
\begin{aligned}
& \lim _{a \rightarrow \infty} \sup _{m \geq 1, n \geq 1} \frac{1}{k_{m n}} \sum_{i=1}^{u_{m}} \sum_{j=1}^{v_{n}} E\left\|k_{m n}^{1 / r} A_{m n i j} V_{i j}\right\|^{r} I\left(\left\|V_{i j}\right\|^{r}>a\right) \\
& \leq \lim _{a \rightarrow \infty} \sup _{m \geq 1, n \geq 1} \sum_{i=1}^{u_{m}} \sum_{j=1}^{v_{n}} E\left\|A_{m n i j} V_{i j}\right\|^{r} I\left(\left\|V_{i j}\right\|>a\right)=0 .
\end{aligned}
$$

This implies

$$
\lim _{a \rightarrow \infty} \sup _{m \geq 1, n \geq 1} \frac{1}{k_{m n}} \sum_{i=1}^{u_{m}} \sum_{j=1}^{v_{n}} E\left\|k_{m n}^{1 / r} A_{m n i j} V_{i j}\right\|^{r} I\left(\left\|V_{i j}\right\|^{r}>a\right)=0 .
$$

By Theorem 3.1 we obtain the proof.

Theorem 3.3. Let $0<r<1$. Suppose that $\left\{V_{m n} ; m \geq 1, n \geq 1\right\}$ satisfies the Cesàro type uniform integrability with exponent $r$, in the sense that (2.1) and (2.2) hold. Then

$$
\frac{1}{k_{m n}^{\frac{1}{r}}} \sum_{i=1}^{u_{m}} \sum_{j=1}^{v_{n}} V_{i j} \rightarrow 0 \text { in } L_{r} \text { as } n \vee m \rightarrow \infty
$$

Proof.

$$
\begin{aligned}
& E\left\|\frac{1}{k_{m n}^{\frac{1}{r}}} \sum_{i=1}^{u_{m}} \sum_{j=1}^{v_{n}} V_{i j}\right\|^{r}=\frac{1}{k_{m n}} E\left\|\sum_{i=1}^{u_{m}} \sum_{j=1}^{v_{n}} V_{i j}\right\|^{r} \\
= & \frac{1}{k_{m n}} E\left\|\sum_{i=1}^{u_{m}} \sum_{j=1}^{v_{n}} V_{i j} I\left(\left\|V_{i j}\right\|^{r} \leq k_{m n}\right)+\sum_{i=1}^{u_{m}} \sum_{j=1}^{v_{n}} V_{i j} I\left(\left\|V_{i j}\right\|>k_{m n}\right)\right\| \\
\leq & \frac{1}{k_{m n}} E\left\|\sum_{i=1}^{u_{m}} \sum_{j=1}^{v_{n}} V_{i j} I\left(\left\|V_{i j}\right\|^{r} \leq k_{m n}\right)\right\|^{r}+\frac{1}{k_{m n}} E\left\|\sum_{i=1}^{u_{m}} \sum_{j=1}^{v_{n}} V_{i j} I\left(\left\|V_{i j}\right\|^{r}>k_{m n}\right)\right\|^{r} \\
\leq & \frac{1}{k_{m n}}\left(E\left\|\sum_{i=1}^{r} \sum_{j=1}^{v_{n}} V_{i j} I\left(\left\|V_{i j}\right\|^{r} \leq k_{m n}\right)\right\|\right)^{u_{i}}
\end{aligned}
$$




$$
\begin{aligned}
& \quad+\frac{1}{k_{m n}} E\left\|\sum_{i=1}^{u_{m}} \sum_{j=1}^{v_{n}} V_{i j} I\left(\left\|V_{i j}\right\|^{r}>k_{m n}\right)\right\|^{r} \quad \text { (by Liapunov's inequality) } \\
& \leq\left(\frac{1}{k_{m n}^{\frac{1}{r}}} \sum_{i=1}^{u_{m}} \sum_{j=1}^{v_{n}} E\left\|V_{i j}\right\| I\left(\left\|V_{i j}\right\|^{r} \leq k_{m n}\right)\right)^{r} \\
& \quad+\left(\sup _{m \geq 1, n \geq 1} \frac{1}{k_{m n}} \sum_{i=1}^{u_{m}} \sum_{j=1}^{v_{n}} E\left\|V_{i j}\right\|^{r} I\left(\left\|V_{i j}\right\|^{r}>k_{m n}\right)\right) \rightarrow 0 \text { as } m \vee n \rightarrow \infty,
\end{aligned}
$$

by Lemma 2.1 with $\beta=1$ and (2.2). The proof is completed.

Corollary 3.4. Let $0<r<1$ and $\left\{A_{m n i j} ; m \geq 1, n \geq 1,1 \leq i \leq u_{m}, 1 \leq j \leq\right.$ $\left.v_{n}\right\}$ be an array of random variables satisfying $\sum_{i=1}^{u_{m}} \sum_{j=1}^{v_{n}} E\left|A_{m n i j}\right|^{r} \leq C<\infty$ for all $m \geq 1, n \geq 1$. Suppose that $A_{m n i j}$ and $V_{i j}$ are independent for all $m \geq 1, n \geq 1,1 \leq i \leq u_{m}, 1 \leq j \leq v_{n}$. Assume that the following conditions hold:

(i) $\left\{\left\|V_{m n}\right\|^{r} ; m \geq 1, n \geq 1\right\}$ is $\left\{E\left|A_{m n i j}\right|^{r}\right\}$-uniformly integrable;

(ii) $\sup _{1 \leq i \leq u_{m}, 1 \leq j \leq v_{n}} E\left|A_{m n i j}\right| \rightarrow 0$ as $m \vee n \rightarrow \infty$.

Then

$$
\sum_{i=1}^{u_{m}} \sum_{j=1}^{v_{n}} A_{m n i j} V_{i j} \rightarrow 0 \text { in } L_{r} \text { as } m \vee n \rightarrow \infty
$$

Proof. The proof is similar to that of Corollary 3.2.

In the following theorem, we establish the weak law of large numbers with random indices for weighted double sums of random elements.

Theorem 3.5. Let $1 \leq r<p \leq 2$, Banach space $\mathcal{X}$ be a martingale type $p$. Suppose that $\left\{V_{m n} ; m \geq 1, n \geq 1\right\}$ satisfies the Cesàro type uniform integrability with exponent $r$, in the sense that (2.1) and (2.2) hold. Let $\left\{T_{n} ; n \geq 1\right\}$ and $\left\{\tau_{n} ; n \geq 1\right\}$ be sequences of positive integer-valued random variables such that

$$
\lim _{n \rightarrow \infty} P\left\{T_{n}>u_{n}\right\}=\lim _{n \rightarrow \infty} P\left\{\tau_{n}>v_{n}\right\}=0 .
$$

Then

$$
\frac{1}{k_{m n}^{\frac{1}{r}}} \sum_{i=1}^{T_{m}} \sum_{j=1}^{\tau_{n}}\left(V_{i j}-E\left(V_{i j} \mid \mathcal{F}_{i j}\right)\right) \stackrel{P}{\longrightarrow} 0 \text { as } n \wedge m \rightarrow \infty .
$$

Proof. For arbitrary $\varepsilon>0$,

$$
P\left\{\frac{1}{k_{m n}^{\frac{1}{r}}}\left\|\sum_{i=1}^{T_{m}} \sum_{j=1}^{\tau_{n}}\left(V_{i j}-E\left(V_{i j} \mid \mathcal{F}_{i j}\right)\right)\right\|>\varepsilon\right\}
$$


MEAN CONVERGENCE THEOREMS AND WLLNS FOR DOUBLE ARRAYS 477

$$
\begin{aligned}
\leq & P\left\{\left(\frac{1}{k_{m n}^{\frac{1}{r}}}\left\|\sum_{i=1}^{T_{m}} \sum_{j=1}^{\tau_{n}}\left(V_{i j}-E\left(V_{i j} \mid \mathcal{F}_{i j}\right)\right)\right\|>\varepsilon\right) \bigcap\left(T_{m} \leq u_{m}\right) \bigcap\left(\tau_{n} \leq v_{n}\right)\right\} \\
& +P\left\{T_{m}>u_{m}\right\}+P\left\{\tau_{n}>v_{n}\right\} .
\end{aligned}
$$

Set the same $V_{m n i j}^{\prime}, V_{m n i j}^{\prime \prime}, U_{m n i j}^{\prime}$ and $U_{m n i j}^{\prime \prime}$ as in the proof of Theorem 3.1 we have

$$
\begin{aligned}
& P\left\{\left(\frac{1}{k_{m n}^{\frac{1}{r}}}\left\|\sum_{i=1}^{T_{m}} \sum_{j=1}^{\tau_{n}}\left(V_{i j}-E\left(V_{i j} \mid \mathcal{F}_{i j}\right)\right)\right\|>\varepsilon\right) \bigcap\left(T_{m} \leq u_{m}\right) \bigcap\left(\tau_{n} \leq v_{n}\right)\right\} \\
& \leq P\left\{\bigcup_{i=1}^{u_{m}} \bigcup_{j=1}^{v_{n}}\left(\frac{1}{k_{m n}^{\frac{1}{r}}}\left\|\sum_{i=1}^{k} \sum_{j=1}^{l}\left(V_{i j}-E\left(V_{i j} \mid \mathcal{F}_{i j}\right)\right)\right\|>\varepsilon\right)\right\} \\
& =P\left\{\frac{1}{k_{m}^{\frac{1}{r}}} \max _{1 \leq k \leq u_{m}} \max _{1 \leq l \leq v_{n}}\left\|\sum_{i=1}^{k} \sum_{j=1}^{l}\left(V_{i j}-E\left(V_{i j} \mid \mathcal{F}_{i j}\right)\right)\right\|>\varepsilon\right\} \\
& \leq P\left\{\frac{1}{k_{m n}^{\frac{1}{r}}} \max _{1 \leq k \leq u_{m}} \max _{1 \leq l \leq v_{n}}\left\|\sum_{i=1}^{k} \sum_{j=1}^{l}\left(V_{m n i j}^{\prime}-U_{m n i j}^{\prime}\right)\right\|>\frac{\varepsilon}{2}\right\} \\
& +P\left\{\frac{1}{k_{m n}^{\frac{1}{r}}} \max _{1 \leq k \leq u_{m}} \max _{1 \leq l \leq v_{n}}\left\|\sum_{i=1}^{k} \sum_{j=1}^{l}\left(V_{m n i j}^{\prime \prime}-U_{m n i j}^{\prime \prime}\right)\right\|>\frac{\varepsilon}{2}\right\} \\
& \leq \frac{C}{\varepsilon^{p} k_{m n}^{\frac{p}{p}}} \sum_{i=1}^{u_{m}} \sum_{j=1}^{v_{n}} E\left\|V_{m n i j}^{\prime}-U_{m n i j}^{\prime}\right\|^{p} \\
& +\frac{C}{\varepsilon^{r} k_{m n}} \sum_{i=1}^{u_{m}} \sum_{j=1}^{v_{n}} E\left\|V_{m n i j}^{\prime \prime}-U_{m n i j}^{\prime \prime}\right\|^{r} \quad \text { (by Lemma 2.3) } \\
& \leq \frac{C}{\varepsilon^{p} k_{m n}^{\frac{p}{p}}}\left(\sum_{i=1}^{u_{m}} \sum_{j=1}^{v_{n}} E\left\|V_{i j}\right\|^{p} I\left(\left\|V_{i j}\right\|^{r} \leq k_{m n}\right)\right) \\
& +\frac{C}{\varepsilon^{r} k_{m n}}\left(\sum_{i=1}^{u_{m}} \sum_{j=1}^{v_{n}} E\left\|V_{i j}\right\|^{r} I\left(\left\|V_{i j}\right\|^{r}>k_{m n}\right)\right) \text { (by } c_{r} \text {-inequality) } \\
& \leq \frac{C}{\varepsilon^{p}}\left(\frac{1}{k_{m n}^{\frac{p}{p}}} \sum_{i=1}^{u_{m}} \sum_{j=1}^{v_{n}} E\left\|V_{i j}\right\|^{p} I\left(\left\|V_{i j}\right\|^{r} \leq k_{m n}\right)\right) \\
& +\frac{C}{\varepsilon^{r}}\left(\sup _{m \geq 1, n \geq 1} \frac{1}{k_{m n}} \sum_{i=1}^{u_{m}} \sum_{j=1}^{v_{n}} E\left\|V_{i j}\right\|^{r} I\left(\left\|V_{i j}\right\|^{r}>k_{m n}\right)\right) \rightarrow 0 \\
& \text { as } m \wedge n \rightarrow \infty \text {, }
\end{aligned}
$$


by Lemma 2.1 with $\beta=p$ and (2.2).

The conclusion (3.2) follows immediately from (3.1), (3.3) and (3.4).

The below corollary is inferred from the above theorem and the proof is similar to that of Corollary 3.2.

Corollary 3.6. Let $1 \leq r<p \leq 2$, Banach space $\mathcal{X}$ be a martingale type $p$. Let $\left\{A_{m n i j} ; m \geq 1, n \geq 1,1 \leq i \leq u_{m}, 1 \leq j \leq v_{n}\right\}$ be an array of random variables satisfying $\sum_{i=1}^{u_{m}} \sum_{j=1}^{v_{n}} E\left|A_{m n i j}\right|^{r} \leq C<\infty$ for all $m \geq 1, n \geq 1$. Suppose that $\left\{A_{m n i j} ; 1 \leq i \leq u_{m}, 1 \leq j \leq v_{n}\right\}$ and $\left\{V_{i j} ; i \geq 1, j \geq 1\right\}$ are independent for all $m \geq 1, n \geq 1$. Let $\left\{T_{n} ; n \geq 1\right\}$ and $\left\{\tau_{n} ; n \geq 1\right\}$ be sequences of positive integervalued random variables satisfying (3.1). Assume that the following conditions hold:

(i) $\left\{\left\|V_{m n}\right\|^{r} ; m \geq 1, n \geq 1\right\}$ is $\left\{E\left|A_{m n i j}\right|^{r}\right\}$-uniformly integrable;

(ii) $\quad \sup E\left|A_{m n i j}\right| \rightarrow 0$ as $m \vee n \rightarrow \infty$.

Then $1 \leq i \leq u_{m}, 1 \leq j \leq v_{n}$

$$
\sum_{i=1}^{T_{m}} \sum_{j=1}^{\tau_{n}} A_{m n i j}\left(V_{i j}-E\left(V_{i j} \mid \mathcal{F}_{i j}\right)\right) \stackrel{P}{\longrightarrow} 0 \text { as } n \wedge m \rightarrow \infty .
$$

Remark 3.7. In the case of $r=p$, the conclusion of Corollaries 3.2 and 3.6 will fail (see Example 4.1 in Section 4). However, if the condition (ii) is replaced by stronger condition: $\sum_{i=1}^{u_{m}} \sum_{j=1}^{v_{n}} E\left|A_{m n i j}\right|^{p}=0$ as $m \vee n \rightarrow \infty$, then the conclusion of Corollary 3.1 is still right when the hypothesis that $1 \leq r<p \leq 2$ is replaced by $1 \leq r \leq p \leq 2$.

In the case of $0<r<1$ we also have the result which is similar to Theorem 3.5 .

Theorem 3.8. Let $0<r<1$. Suppose that $\left\{V_{m n} ; m \geq 1, n \geq 1\right\}$ satisfies the Cesàro type uniform integrability with exponent $r$, in the sense that (2.1) and (2.2) hold. Let $\left\{T_{n} ; n \geq 1\right\}$ and $\left\{\tau_{n} ; n \geq 1\right\}$ be sequences of positive integervalued random variables satisfying (3.1). Then

$$
\frac{1}{k_{m n}^{\frac{1}{r}}} \sum_{i=1}^{T_{m}} \sum_{j=1}^{\tau_{n}} V_{i j} \stackrel{P}{\longrightarrow} 0 \text { as } n \wedge m \rightarrow \infty .
$$

Corollary 3.9. Let $0<r<1$. Let $\left\{A_{m n i j} ; m \geq 1, n \geq 1,1 \leq i \leq u_{m}, 1 \leq j \leq\right.$ $\left.v_{n}\right\}$ be an array of random variables satisfying $\sum_{i=1}^{u_{m}} \sum_{j=1}^{v_{n}} E\left|A_{m n i j}\right|^{r} \leq C<\infty$ for all $m \geq 1, n \geq 1$. Suppose that $A_{m n i j}$ and $V_{i j}$ are independent for all $m \geq 1, n \geq 1,1 \leq i \leq u_{m}, 1 \leq j \leq v_{n}$. Let $\left\{T_{n} ; n \geq 1\right\}$ and $\left\{\tau_{n} ; n \geq 1\right\}$ be sequences of positive integer-valued random variables satisfying (3.1). Assume that the following conditions hold:

(i) $\left\{\left\|V_{m n}\right\|^{r} ; m \geq 1, n \geq 1\right\}$ is $\left\{E\left|A_{m n i j}\right|^{r}\right\}$-uniformly integrable;

(ii) $\sup _{1 \leq i \leq u_{m}, v_{n}} E\left|A_{m n i j}\right| \rightarrow 0$ as $m \vee n \rightarrow \infty$. 
Then

$$
\sum_{i=1}^{T_{m}} \sum_{j=1}^{\tau_{n}} A_{m n i j} V_{i j} \stackrel{P}{\longrightarrow} 0 \text { as } m \vee n \rightarrow \infty
$$

\section{Some interesting examples}

Four illustrative examples will now be presented. For $1 \leq q \leq 2$, let $l_{q}$ denote the Banach space of absolute $q^{t h}$ power summable real sequence $v=\left\{v_{i}, i \geq 1\right\}$ with norm $\|v\|=\left(\sum_{i=1}^{\infty}\left|v_{i}\right|^{q}\right)^{1 / q}$. The element having $\alpha(\alpha \in \mathbb{R})$ in its $k^{t h}$ position and 0 elsewhere will be denoted by $v_{\alpha}^{(k)}, k \geq 1$. Let $\varphi: \mathbb{N} \times \mathbb{N} \rightarrow \mathbb{N}$ be one-to-one and onto map, where $\mathbb{N}=\{1,2,3, \ldots\}$ is the set of all positive integers.

The first example shows that the hypothesis that $1 \leq r<p \leq 2$ in Theorem 3.1 can not be replaced by the hypothesis that $1 \leq r \leq p \leq 2$.

Example 4.1. Let $r=p=2$ and consider the Banach space $l_{2}$ and the array of independent random elements $\left\{V_{i j} ; i \geq 1, j \geq 1\right\}$ in $l_{2}$ with

$$
P\left\{V_{i j}=v_{1}^{(\varphi(i, j))}\right\}=P\left\{V_{i j}=-v_{1}^{(\varphi(i, j))}\right\}=\frac{1}{2}, i, j \geq 1 .
$$

Then $\left\|V_{i j}\right\|=1$ a.s., therefore, (2.2) is automatic. Suppose that $u_{m}=m$, $v_{n}=n$ and $k_{m n}=m n$. Then $(2.1)$ holds since

$$
\sup _{m \geq 1, n \geq 1} \frac{1}{k_{m n}} \sum_{i=1}^{u_{m}} \sum_{j=1}^{v_{n}} E\left\|V_{i j}\right\|^{2}=1 .
$$

Moreover, with probability 1

$$
\frac{1}{k_{m n}}\left\|\sum_{i=1}^{u_{m}} \sum_{j=1}^{v_{n}} V_{i j}\right\|^{2}=1
$$

Thus, the conclusion of Theorem 3.1 fails.

The next example illustrates the essential role that the condition (2.1) plays in Theorem 1.

Example 4.2. Let $3 / 2<p \leq 2, r=2 p / 3, u_{n}=v_{n}=n^{2}, k_{m n}=(m n)^{\beta}$ with $\beta \in \mathbb{N}$ and consider the Banach space $l_{p}$ and the array of independent random elements $\left\{V_{i j} ; i \geq 1, j \geq 1\right\}$ in $l_{p}$ with

$$
P\left\{V_{i j}=v_{1}^{(\varphi(i, j))}\right\}=P\left\{V_{i j}=-v_{1}^{(\varphi(i, j))}\right\}=\frac{1}{2}, i, j \geq 1 .
$$

Then $\left\|V_{i j}\right\|=1$ a.s., therefore, (2.2) is automatic.

If $\beta \geq 2$, then (2.1) holds since

$$
\sup _{m \geq 1, n \geq 1} \frac{1}{k_{m n}} \sum_{i=1}^{u_{m}} \sum_{j=1}^{v_{n}} E\left\|V_{i j}\right\|^{r}=\sup _{m \geq 1, n \geq 1}(m n)^{2-\beta} \leq 1 .
$$


Hence,

$$
\frac{1}{k_{m n}^{\frac{1}{r}}}\left\|\sum_{i=1}^{u_{m}} \sum_{j=1}^{v_{n}} V_{i j}\right\| \rightarrow 0 \text { in } L_{r} \text { as } m \vee n \rightarrow \infty
$$

On the other hand, if $\beta=1$, then

$$
\sup _{m \geq 1, n \geq 1} \frac{1}{k_{m n}} \sum_{i=1}^{u_{m}} \sum_{j=1}^{v_{n}} E\left\|V_{i j}\right\|^{r}=\sup _{m \geq 1, n \geq 1}(m n)=\infty,
$$

whence (2.1) fails. Moreover, with probability 1

$$
\frac{1}{k_{m n}}\left\|\sum_{i=1}^{u_{m}} \sum_{j=1}^{v_{n}} V_{i j}\right\|^{r}=(m n)^{\frac{2 r}{p}-1}=(m n)^{\frac{1}{3}} \geq 1,
$$

and so the conclusion of Theorem 3.1 fails.

The third example shows that in Theorems 3.1 the condition (2.2) cannot be dispensed with.

Example 4.3. Let $r=1, p=2, u_{n}=v_{n}=n, k_{m n}=2^{m} 2^{n}$ and consider the Banach space $l_{2}$ and the array of independent random elements $\left\{V_{i j} ; i \geq 1, j \geq\right.$ 1\} in $l_{2}$ with

$$
P\left\{V_{i j}=v_{\alpha_{i j}}^{(\varphi(i, j))}\right\}=P\left\{V_{i j}=-v_{\alpha_{i j}}^{(\varphi(i, j))}\right\}=\frac{1}{2}, i, j \geq 1,
$$

where $\alpha_{i j}=2^{(i-1)} 2^{(j-1)}$. Then $\left\|V_{i j}\right\|=2^{(i-1)} 2^{(j-1)}$ a.s.. Therefore, (2.1) holds since

$$
\begin{aligned}
\sup _{m \geq 1, n \geq 1} \frac{1}{k_{m n}} \sum_{i=1}^{u_{m}} \sum_{j=1}^{v_{n}} E\left\|V_{i j}\right\|^{r} & =\sup _{m \geq 1, n \geq 1} \frac{1}{2^{m} 2^{n}} \sum_{i=1}^{m} \sum_{j=1}^{n} 2^{(i-1)} 2^{(j-1)} \\
& =\sup _{m \geq 1, n \geq 1} \frac{\left(2^{m}-1\right)\left(2^{n}-1\right)}{2^{m} 2^{n}} \leq 1
\end{aligned}
$$

However, (2.2) fails since with $a=2^{(m+n-4)} \rightarrow \infty$ as $m \vee n \rightarrow \infty$ but

$$
\frac{1}{k_{m n}} \sum_{i=1}^{u_{m}} \sum_{j=1}^{v_{n}} E\left\|V_{i j}\right\|^{r} I\left(\left\|V_{i j}\right\|^{r}>2^{m+n-4}\right)=\frac{2^{m-1} 2^{n-1}}{2^{m} 2^{n}}=\frac{1}{4} \nrightarrow 0 .
$$

Moreover, with probability 1

$$
\begin{aligned}
\frac{1}{k_{m n}}\left\|\sum_{i=1}^{u_{m}} \sum_{j=1}^{v_{n}} V_{i j}\right\|^{r} & =\frac{1}{2^{m} 2^{n}}\left(\sum_{i=1}^{m} \sum_{j=1}^{n} 4^{i-1} 4^{j-1}\right)^{1 / 2} \\
& =\frac{1}{2^{m} 2^{n}}\left(\frac{\left(4^{m}-1\right)\left(4^{n}-1\right)}{9}\right)^{1 / 2} \\
& =\frac{1}{3}\left(\frac{\left(4^{m}-1\right)\left(4^{n}-1\right)}{4^{m} 4^{n}}\right)^{1 / 2} \geq \frac{1}{4}
\end{aligned}
$$


so the conclusion of Theorem 3.1 fails.

Apropos of Theorem 3.3, the last example shows that its hypotheses do not guarantee that the convergence in mean of order $r$ prevails in the conclusion (3.2).

Example 4.4. Let $1 \leq r<p \leq 2$ and consider the Banach space $l_{p}$ and the array of independent random elements $\left\{V_{i j} ; i \geq 1, j \geq 1\right\}$ in $l_{p}$ with

$$
P\left\{V_{i j}=v_{1}^{(\varphi(i, j))}\right\}=P\left\{V_{i j}=-v_{1}^{(\varphi(i, j))}\right\}=\frac{1}{2}, i, j \geq 1 .
$$

Suppose that $u_{n}=v_{n}=2^{n}, k_{m n}=2^{m} 2^{n}$. Then, it is easy to see that $(2.1)$ and (2.2) hold. Let $\left\{T_{n}, n \geq 1\right\}$ and $\left\{\tau_{n}, n \geq 1\right\}$ be sequences of identically distributed random variables with the distribution of $T_{1}$ and $\tau_{1}$ given by

$$
P\left\{T_{1}=2^{i}\right\}=P\left\{\tau_{1}=2^{i}\right\}=2^{-i}
$$

and suppose that $\left\{T_{n} ; n \geq 1\right\}$ is independent of $\left\{\tau_{n} ; n \geq 1\right\}$. Then (3.1) holds since

$$
P\left\{T_{n}>u_{n}\right\}=P\left\{\tau_{n}>v_{n}\right\}=\sum_{i=n+1}^{\infty} 2^{-i} \rightarrow 0 \text { as } n \rightarrow \infty .
$$

Thus, by Theorem 3.3,

$$
\frac{1}{k_{m n}^{\frac{1}{r}}} \sum_{i=1}^{T m} \sum_{j=1}^{\tau_{n}} V_{i j} \stackrel{P}{\longrightarrow} 0 \text { as } n \wedge m \rightarrow \infty
$$

However,

$$
E\left\|\frac{1}{k_{m n}^{\frac{1}{r}}} \sum_{i=1}^{T m} \sum_{j=1}^{\tau_{n}} V_{i j}\right\|^{r}=\frac{E\left(T_{m} \tau_{n}\right)^{r}}{2^{m} 2^{n}}=\frac{E\left(T_{m}\right)^{r} E\left(\tau_{n}\right)^{r}}{2^{m} 2^{n}}=\infty .
$$

\section{References}

[1] A. Adler, A. Rosalsky, and A. I. Volodin, A mean convergence theorem and weak law for arrays of random elements in martingale type $p$ Banach spaces, Statist. Probab. Lett. 32 (1997), no. 2, 167-174.

[2] T. K. Chandra, Uniform integrability in the Cesaro sense and the weak law of large numbers, SankhyāSer. A 51 (1989), no. 3, 309-317.

[3] J. Hoffmann-Jørgensen and G. Pisier, The law of large numbers and the central limit theorem in Banach spaces, Ann. Probability 4 (1976), no. 4, 587-599.

[4] D. Landers and L. Rogge, Laws of large numbers for pairwise independent uniformly integrable random variables, Math. Nachr. 130 (1987), 189-192.

[5] M. Ordóñez Cabrera, Convergence of weighted sums of random variables and uniform integrability concerning the weights, Collect. Math. 45 (1994), no. 2, 121-132.

[6] G. Pisier, Martingales with values in uniformly convex spaces, Israel J. Math. 20 (1975), no. 3-4, 326-350.

7] _ Probabilistic methods in the geometry of Banach spaces, Probability and analysis (Varenna, 1985), 167-241, Lecture Notes in Math., 1206, Springer, Berlin, 1986.

[8] F. S. Scalora, Abstract martingale convergence theorems, Pacific J. Math. 11 (1961), $347-374$. 
[9] S. H. Sung, Weak law of large numbers for arrays of random variables, Statist. Probab. Lett. 42 (1999), no. 3, 293-298.

[10] L. V. Thanh, Mean convergence theorems and weak laws of large numbers for double arrays of random variables, J. Appl. Math. Stoch. Anal. 2006 (2006), Art. ID 49561, $15 \mathrm{pp}$.

Le Van Dung

FACUlTy of Mathematics

DANANG UNIVERSity OF EdUCATION

459 Ton Duc Thang, Danang, Vietnam

E-mail address: lvdunght@gmail.com

Nguyen Duy Tien

Faculty of Mathematics

NATIONAL UNiVERSity OF HANOI

334 Nguyen Trai, Hanoi, Vietnam

E-mail address: nduytien2006@yahoo.com 